\title{
Relations entre différents dépôts gras et éléments de peau chez le poulet
}

\author{
F.H. RICARD \\ avec la collaboration technique de G. MARCHE \\ I.N.R.A., Station de Recherches avicoles \\ Nouzilly, F 37380 Monnaie
}

\begin{abstract}
Résumé
Les dépôts gras abdominaux, les dépôts gras sous-cutanés des cuisses et pilons, dı cou et du reste de la carcasse, les dépôts gras inter-musculaires des cuisses et pilons, ainsi que la peau des cuisses et pilons, du cou, du dos et du reste de la carcasse ont été disséqués sur deux échantillons de poulets de type différent.

La comparaison des deux échantillons montre que les poulets les plus gras développent relativement plus les dépôts gras abdominaux et sous-cutanés que les dépôts gras intermusculaires. Ils ont aussi un pourcentage de peau un peu plus élevé.

Les corrélations ont été calculées, d'une part entre les poids bruts de chaque élément disséqué, et d'autre part, entre les valeurs exprimées en pour cent du poids vif. Le poids vif est faiblement corrélé avec le poids des dépôts gras abdominaux et sous-cutanés. Les corrélations entre poids des différents dépôts gras varient de 0,4 à 0,9 ; les corrélations entre poids de peau varient de 0,1 à 0,9 ; les corrélations entre poids de dépôts gras et poids de peau varient de 0,1 à 0,5 .

Le poids du fragment de pcau prélevé sur le dos présente des corrélations souvent faibles avec les autres variables et ne constitue pas une bonne variable prédictrice. Au contraire, le dépôt gras sous-cutané et la peau disséqués au niveau des cuisses et pilons sont fortement corrélés $(0,9)$ avec les dépôts gras sous-cutanés et la peau de l'ensemble de la carcasse.
\end{abstract} poulet.

Mots clés : gras abdominal, gras sous-cutané, gras intermusculaire, peau, corrélations,

\section{Introduction}

La composition corporelle des volailles fait l'objet actuellement de nombreux travaux, du fait de l'importance que prennent les problèmes d'engraissement et de rendement en viande. Chez le poulet, de nombreux essais ont été réalisés pour estimer l'état d'engraissement. Ils ont été passés en revue récemment (RICARD, 1983). On se base le plus souvent sur la dissection des dépôts gras abdominaux (Delpech \& RICARD, 1965), mais certains auteurs ont proposé d'utiliser les caractéristiques de la peau. C'est ainsi que Moran, Summers \& ORR (1968) ont proposé le dosage des 
lipides d'un triangle de la peau du dos prélevé entre les apophyses ilio-fémorales et la glande uropygienne. Chez le canard et la dinde, c'est le développement de la peau et du gras sous-cutané qui pose un problème aux industriels de la découpe (Thery, 1983).

L'estimation de la composition corporelle à partir d'un seul paramètre, le poids du gras abdominal ou la teneur en lipides de la peau, ne rend pas compte des variations possibles de la répartition sur la carcasse des différents dépôts gras ou des autres tissus. Or, une telle variation existe quand on compare des animaux de type différent. Ainsi, la comparaison d'une lignée grasse et d'une lignée maigre, obtenues par une sélection divergente sur l'importance du dépôt gras abdominal, a montré que si l'écart maximum s'obtenait pour ce dernier (coefficient 4), les dépôts gras sous-cutanés (coefficient 2,8 ) et inter-musculaires (coefficient 1,5 ) donnaient des différences moins accusées, la teneur en lipides du muscle lui-même restant inchangée (Ricard, Leclerco \& Touraille, 1983). La comparaison des lignées grasse et maigre de LiLburn et al. (1982) donne des résultats analogues. Griffiths, Leeson \& Summers (1978), de même que Scheele, Vanschagen \& Ten Have (1981), observent que les différences génétiques sont plus importantes pour le gras abdominal que pour les autres dépôts gras.

Ces observations nous ont conduit à rechercher les relations pouvant exister entre les différents dépôts gras et les parties de peau qu'il est possible de disséquer de façon simple sur la carcasse du poulet, puis à discuter de leur utilisation pratique éventuelle.

\section{Matériel et méthodes}

\section{A. Matériel animal}

L'expérience a porté sur deux échantillons de poulets de type différent, abattus à des âges et à des poids correspondant à leur utilisation commerciale :

- Echantillon A : 16 coquelets d'un croisement Cornish $\times$ White-Rock, donnant des poulets à croissance rapide, abattus à l'âge de 8 semaines. Entre 0 et 4 semaines, ils avaient reçu un aliment dosant $3020 \mathrm{Kcal}$ d'énergie métabolisable et $220 \mathrm{~g}$ de matières azotées totales par $\mathrm{kg}$. Entre 4 et 8 semaines, l'aliment contenait respectivement $3100 \mathrm{Kcal}$ et $195 \mathrm{~g}$ de M.A.T.

- Echantillon B : 18 coquelets d'une souche pure synthétique, utilisée pour la production de poulets «label», abattus à l'âge de 11 semaines $1 / 2$. Ils avaient reçu le même aliment de l'éclosion à l'abattage, aliment dosant $3100 \mathrm{Kcal}$ d'E.M. et $217 \mathrm{~g}$ de M.A.T. L'origine et les caractéristiques de carcasse de cette souche, appelée I-66, ont été décrites précédemment (RICARD, 1974).

\section{B. Variables étudiées}

- Les animaux ont été pesés immédiatement avant l'abattage, après un jeûne complet d'environ 16 heures. 
- On a disséqué les dépôts gras abdominaux. Il s'agit du tissu gras qui se trouve entre la paroi abdominale et les intestins, se prolonge autour du cloaque et entoure le gésier ainsi que le ventricule succenturié (RICARD, 1983).

- Sur les deux cuisses et pilons, on a disséqué la peau proprement dite, le tissu gras sous-cutané (en y incluant le gras sartorial), ainsi que les dépôts gras situés entre les muscles (gras inter-musculaire).

- Le cou a été sectionné au ras des épaules et la tête coupée à la jonction crâne atlas. On a disséqué la peau du cou et les dépôts gras sous-cutanés.

- Après avoir découpé les cuisses et pilons, on a prélevé la peau du dos restant attachée aux os du bassin, limitée à la glande uropygienne. Ce morceau de peau a la forme d'un losange irrégulier que nous avons appelé «écusson». On peut considérer que ses caractéristiques (à l'exception de la forme) sont analogues à celles du triangle de peau défini par Moran, Summers \& Orr (1968).

- Sur tout ce qui reste de la carcasse, ailes exceptées, on a également prélevé la peau et les dépôts gras sous-cutanés.

Par dépôts gras sous-cutanés, nous entendons les dépôts situés entre la peau et les muscles, prélevés sans endommager le derme de la peau.

Chaque élément disséqué a été pesé au décigramme. On a ensuite calculé le poids total des dépôts gras sous-cutanés et le poids total de la peau. Chaque élément a également été exprimé en p. 100 du poids vif avant abattage. Nous avons ainsi obtenu les 23 variables qui sont indiquées dans le tableau 1.

\section{Calculs}

Les paramètres statistiques (moyenne et écart-type) ont été calculés séparément pour chacun des deux échantillons. L’homogénéité des variances a été testée en calculant le rapport de la plus grande à la plus petite. Les moyennes ont été comparées par le test $\mathrm{t}$ de Student.

Les corrélations phénotypiques entre variables ont été calculécs séparément pour chaque échantillon, d'une part pour l'ensemble des valeurs brutes (poids), d'autre part pour l'ensemble des éléments de dissection exprimés en p. $100 \mathrm{du}$ poids vif. Les valeurs des corrélations obtenues pour les deux échantillons ont été comparées et une valeur commune calculée, en passant par l'intermédiaire de la variable $\mathrm{z}$ de Fisher. La valeur commune a été considérée significativement différente de 0 lorsque l'intervalle de confiance (calculé sur la transformée z) ne comprenait pas la valeur 0 (DAGnelie, 1975).

\section{Résultats}

Le tableau 1 donne la moyenne et l'écart-type des 23 variables étudiées, séparément pour chacun des deux échantillons, ainsi que les éléments de la comparaison des échantillons : rapport des moyennes, rapport des variances, test $t$ de compa- 


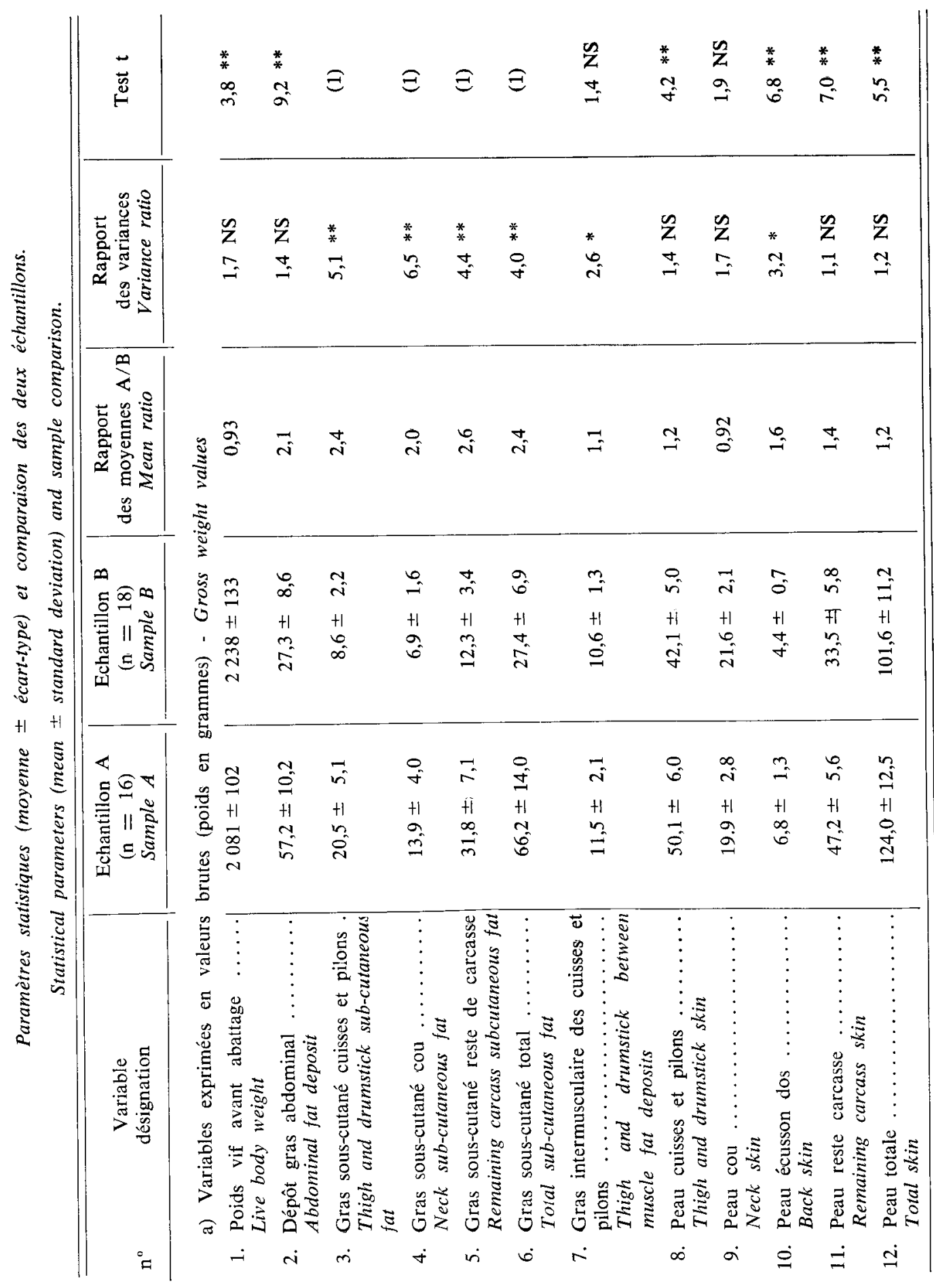




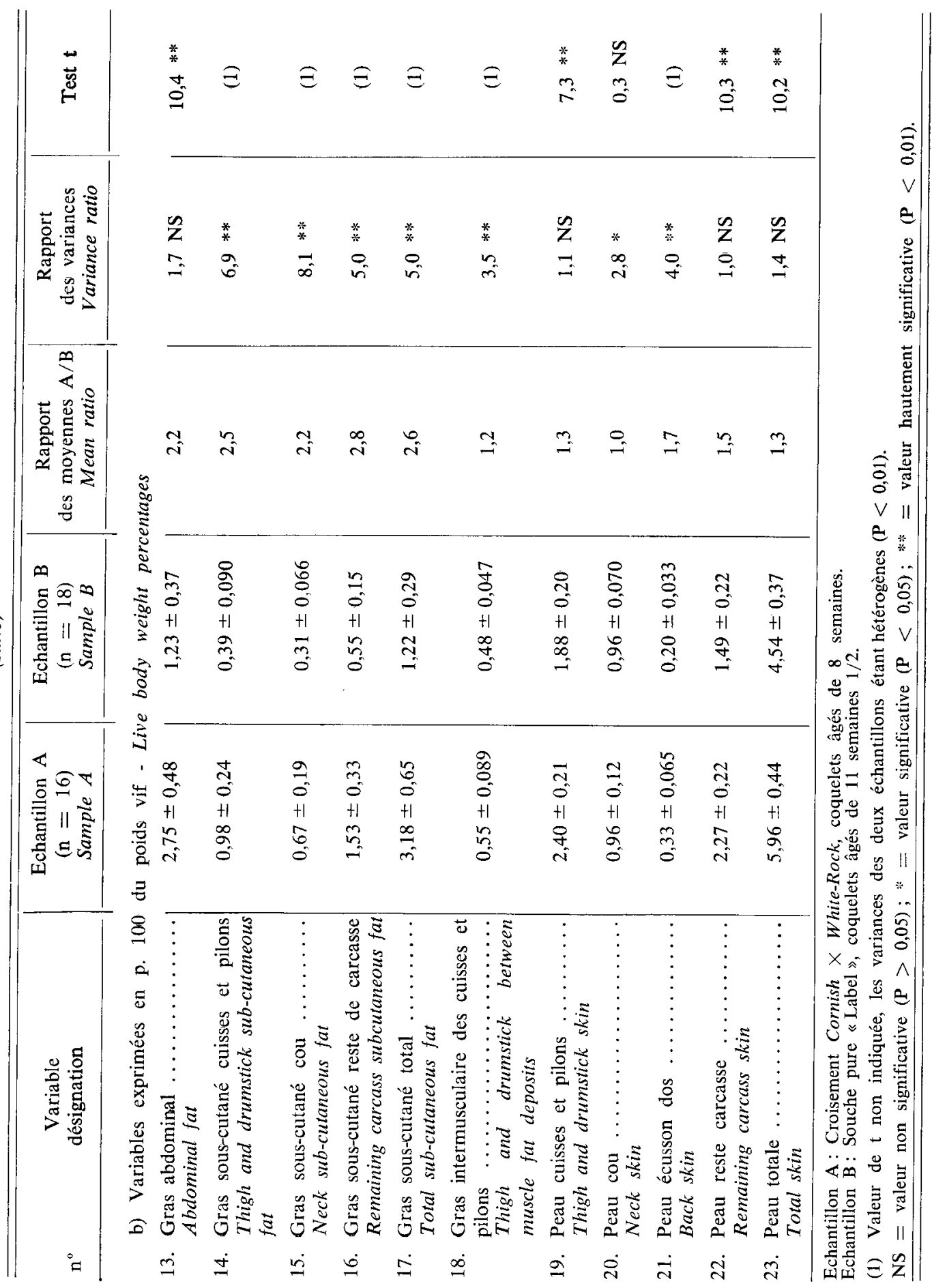




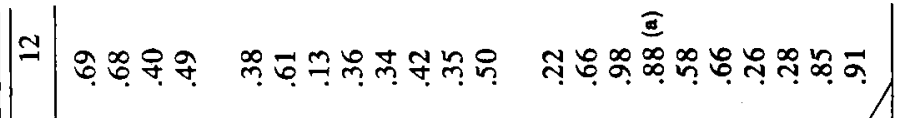
=|ที丶万์

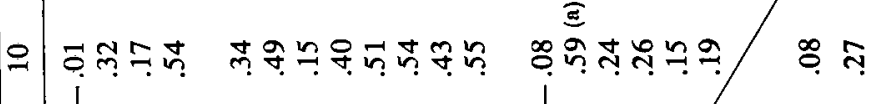

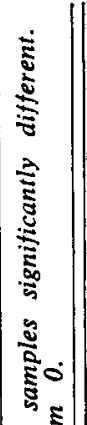

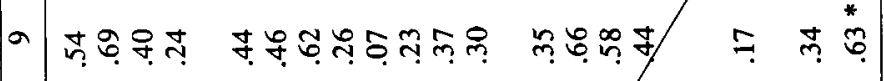

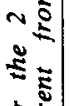

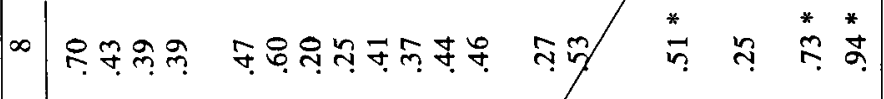
दे

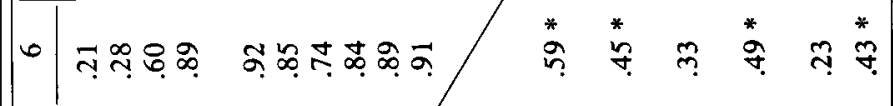
จ इ

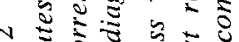

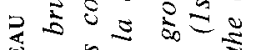

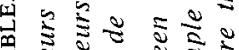

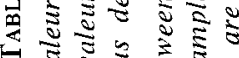
$\Xi \geq \approx$

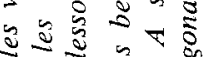

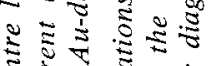

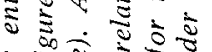

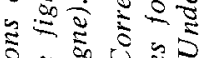

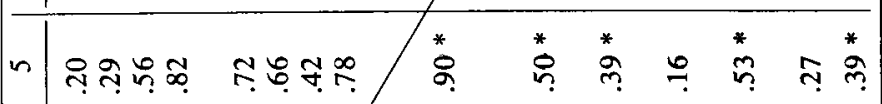

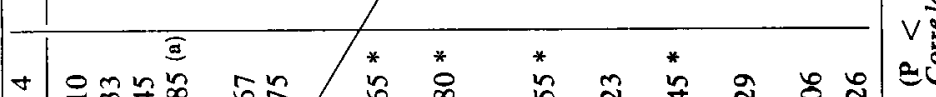

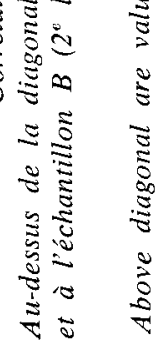
+ ๆ

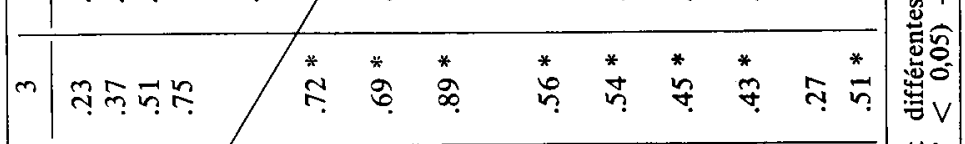
ง

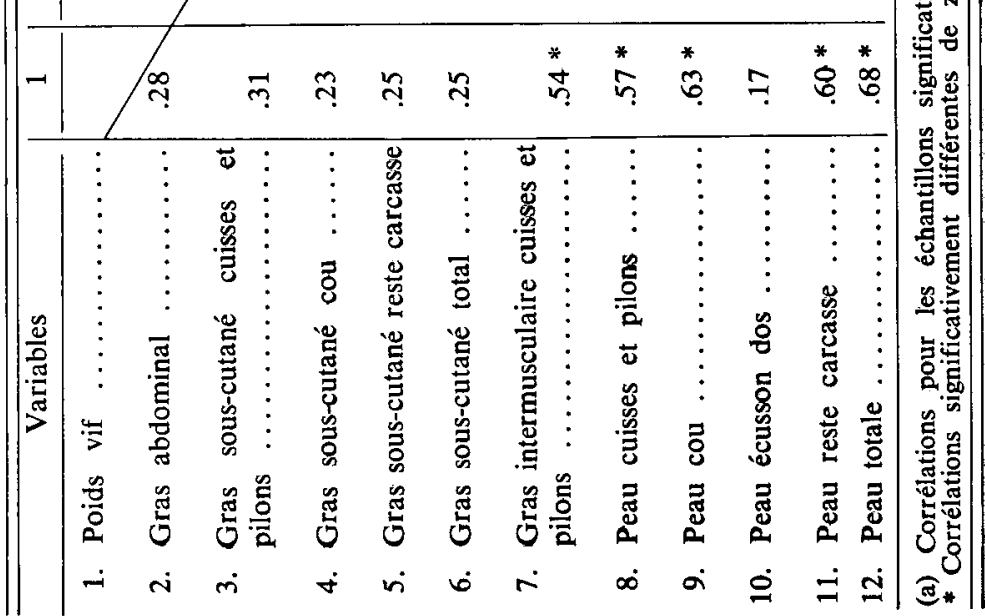


raison des moyennes. Pour certaines variables, en particulier celles correspondant aux dépôts gras sous-cutanés, les variances ne sont pas homogènes; la valeur de $t$ n'a pas été indiquée quand cette hétérogénéité était significative au seuil de 1 p. 100.

Le tableau 1 montre que les deux échantillons correspondent à des animaux de type différent : les poulets A sont significativement plus légers et plus gras (au vu du pourcentage du gras abdominal, Delpech \& Ricard, 1965) que les poulets B. Mais le type génétique et l'alimentation reçue ne sont pas les mêmes.

Les corrélations entre variables exprimées en valeurs brutes (numérotées de 1 à 12) sont indiquées dans le tableau 2. Les corrélations entre variables exprimées en p. $100 \mathrm{du}$ poids vifs (numérotées de 13 à 23) figurent dans le tableau 3. Bien que les deux types de poulets soient différents, le nombre de cas où les deux corrélations sont significativement différentes est faible ( 3 cas sur 66 pour le tableau 2 et 2 cas sur 55 pour le tableau 3 ). On peut donc considérer que les valeurs communes (qui figurent sous la diagonale des tableaux 2 et 3) représentent une bonne estimation de ces corrélations chez le poulet.

On constate (tabl. 2) que le poids vif est faiblement corrélé au poids des dépôts gras abdominaux et sous-cutanés (aucune valeur significative). La corrélation avec le poids des dépôts gras inter-musculaires est plus élevée (de l'ordre de 0,5 ), de même que les corrélations avec les poids de peau, à l'exception de la variable «peau-écusson dos ». Les corrélations entre poids des différents dépôts gras sont assez fortes $(0,4$ à 0,9$)$. Les corrélations entre différents poids de peau sont un peu moins élevées mais significativement différentes de 0 , à l'exception de celles faisant intervenir la variable "peau-écusson dos". Les corrélations entre variables « gras » et variables « peau » sont relativement peu élevées, de 0,1 à 0,5.

Les corrélations entre le poids vif et les variables exprimées en p. $100 \mathrm{du}$ poids vif sont faibles et non significatives. Entre pourcentages, on observe les mêmes tendances que pour les corrélations entre valeurs brutes (tabl. 3).

\section{Discussion}

La différence d'engraissement entre les deux échantillons peut s'expliquer par le type de sélection et l'aliment qu'ils ont reçu. Les poulets A sont issus de souches sélectionnées pour une forte vitesse de croissance, ce qui favorise l'engraissement (LiN, 1981), alors que les poulets B appartiennent à une souche sélectionnée pour une croissance modérée. D'autre part, les poulets $B$ ont reçu, après 4 semaines, un aliment plus riche en matières azotées que les poulets $A$, ce qui est un facteur de diminution de l'engraissement (voir par exemple le travail de Touchburn, Simon \& LeCLERCQ, 1981).

Cette différence d'engraissement permet de rapprocher les résultats présentés ici à ceux de la comparaison des lignées grasse et maigre faite récemment (RICARD, Leclerco \& Touraille, 1983). Les différences étaient plus importantes en ce qui concerne les dépôts gras sous-cutanés et, surtout, le dépôt gras abdominal. Mais nous retrouvons le fait que la différence concernant les dépôts gras inter-musculaires est nettement plus faible (rapport des moyennes de 1,1) que celles concernant les autres 


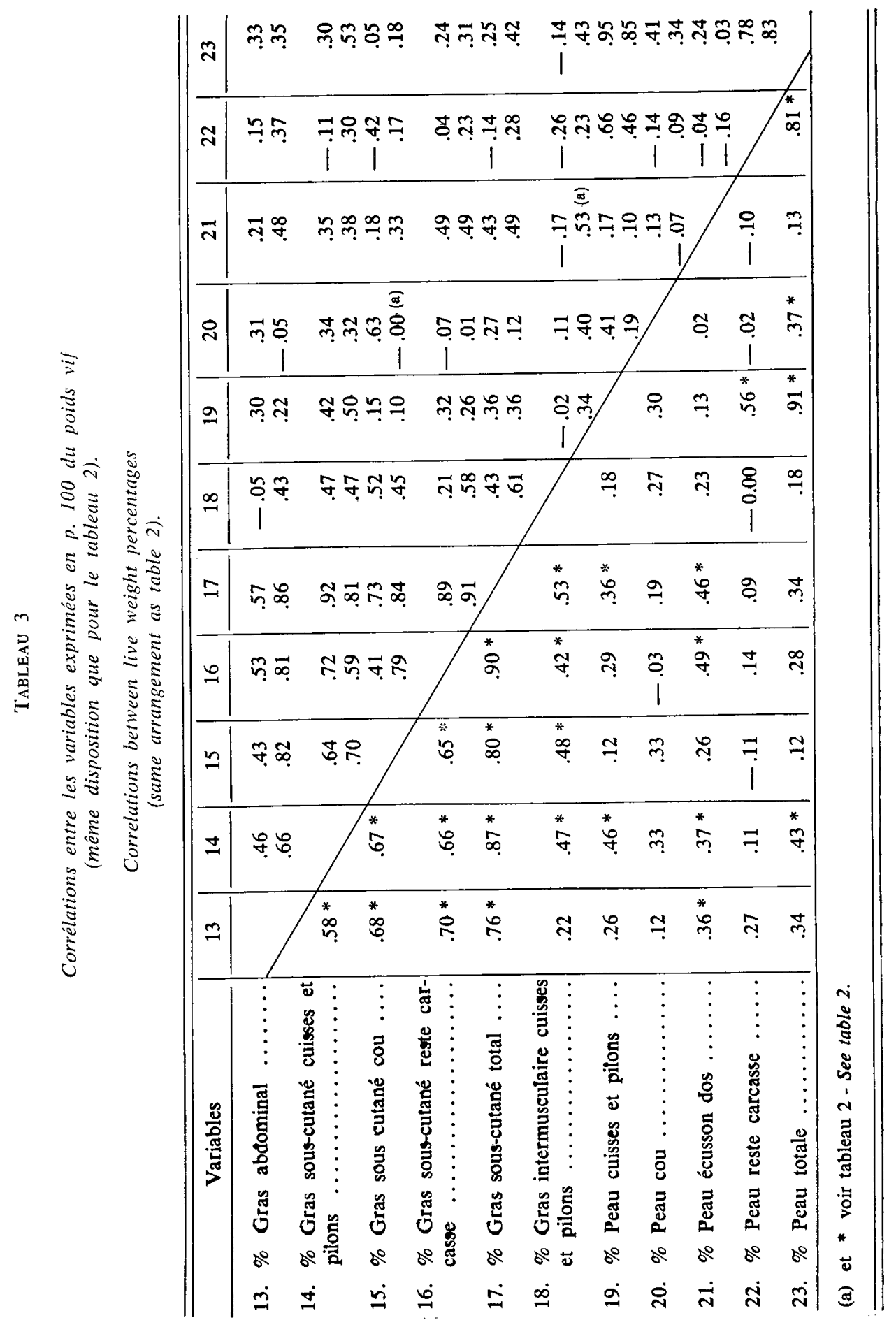


dépôts (rapport des moyennes supérieur à 2). La présente expérience confirme donc que les souches génétiquement grasses développent relativement plus leurs dépôts gras abdominaux et sous-cutanés.

Les corrélations figurant dans les tableaux 2 et 3 sont généralement du même ordre de grandeur que les quelques valeurs trouvées dans la littérature. Dès 1937, GUTTERIDGE avait noté une corrélation positive significative entre le gras abdominal et le gras sous-cutané. Becker et al. (1979) ont observé une corrélation de l'ordre de 0,5 entre le gras abdominal, exprimé en p. 100 du poids vif, et la teneur en lipides d'un morceau de peau du dos. Burgener, Cherry \& Siegel (1981) signalent une corrélation de 0,7 entre le dépôt gras abdominal et le dépôt gras attaché au muscle sartorius (appelé gras sartorial), dépôt qu'ils estiment susceptible d'être mesuré par simple biopsie. En ce qui concerne la peau, nous avions observé dans d'autres souches des corrélations du même ordre de grandeur (RICARD, 1968, 1972).

Les corrélations indiquées dans les tableaux 2 et 3 sont relativement faibles quand elles font intervenir les variables "peau-écusson dos " ou peau du cou et peau du reste de carcasse (corrélations entre pourcentages). Une raison possible est la relative imprécision qu'on peut attacher à la dissection de ces éléments. De ce point de vue, le poids d'un morceau de peau prélevé sur le dos du poulet ne semble pas devoir être retenu comme une bonne variable prédictrice de l'importance du compartiment peau, contrairement à la peau des cuisses et pilons (qui représente la plus forte proportion du poids total de la peau).

Les valeurs moyennes des corrélations observées entre mesures de gras et mesures de peau laissent penser que le poids de la peau débarrassée de son gras souscutané est un caractère relativement peu lié à l'engraissement. Il est, par contre, plus lié au poids vif, mais à l'intérieur d'un même échantillon : les poulets B sont plus lourds, mais ils ont un pourcentage de peau plus faible que les poulets $A$.

En ce qui concerne les dépôts gras sous-cutanés, ce sont les dépôts des cuisses et pilons d'une part, du reste de la carcasse d'autre part, qui donnent les corrélations les plus élevées, de l'ordre de 0,9. Mais d'un point de vue pratique, il est plus simple et plus rapide de disséquer la cuisse et le pilon que le reste de la carcasse. Ces résultats confirment l'observation déjà faite (RICARD, 1972) selon laquelle la dissection d'une cuisse et d'un pilon permet une très bonne estimation de la composition corporelle de l'ensemble de la carcasse du poulet.

\section{Summary}

\section{Relationships between fat deposits and skin regions in broiler chicken carcasses}

\footnotetext{
Abdominal, sub-cutaneous, thigh and drumstick intermuscular fat deposits, as well as thigh and drumstick, neck, back and remaining carcass skin were dissected in two different samples of chickens. The first one (sample A) consisted of 16 cockerels from a commercial Cornish $\mathrm{x}$ White Rock cross slaughtered at 8 weeks of age, while the second one (sample B) was composed of 18 cockerels from a label strain slaughtered at $111 / 2$ weeks of age.

The A cockerels were lighter and fatter than the B ones (tabl. 1). The fatter birds showed relatively more abdominal and sub-cutaneous fat than between muscle fat deposits. They had a higher skin percentage.
} 
Correlations were computed separately between raw weight values (tabl. 2) and for liveweight percentages (tabl. 3) of dissected carcass components. Low and non significant values were obtained for correlations between live body weight and abdominal or sub-cutaneous fat weights. The correlations between the different sites of fat deposition varied from 0.4 to 0.9 , while the correlations between the weights of the various skin regions ranged from 0.1 to 0.9 . Correlations between fat deposits and skin weights ranged from 0.1 to only 0.5 .

The weight of the back skin piece was poorly correlated with the other dissected components; back skin weight is therefore not a good indicator of skin development. On the contrary, thigh and drumstick dissection gave an accurate estimate (correlations of .9) of total sub-cutaneous fat and total skin of the broiler carcasses. chicken.

Key words : abdominal fat, sub-cutaneous fat, between muscle fat, skin, correlations

\section{Remerciements}

La présente étude a été faite dans le cadre du projet $n^{\circ} 280$ de la Délégation ciénúrale à la Recherche Scientifique et Technique (contrat C-1297).

Reçu en septembre 1983.

Accepté en ianvier 1984.

\section{Références bibliographiques}

Becker W.A., Sipencer J.V., Mirosh L.W., Verstrate J.A., 1979. Prediction of fat and fat frec live weight in broiler chickens using backskin fat, and live body weight. Poultry Sci., 58, 835-842.

Burgener J.A., Cherry J.A., Siegel P.B., 1981. The association between sartorial fat and fat deposition in meat-type chickens. Poultry Sci., 60, 54-62.

Dagnelie P., 1975. Théorie et méthodes statistiques. Vol. II. Les méthodes de l'inférence statistique. 2" éd., Presses Agronomiques Gembloux, 309-324.

Delpech P., Ricard F.H., 1965. Relation entre les dépôts adipeux viscéraux et les lipides corporels chez le poulet. Ann. Zootech., 14, 181-189.

Griffiths L., Leeson S., Summers J.D., 1978. Studies on abdominal fat with four commercial strains of male broiler chicken. Poultry Sci., 57, 1198-1203.

Gutteridge H.S., 1937. Methods and rations for fattening poultry. Sci. Agric., 17, 340-358.

LiLburn M.S., Leach L.M. Jr., Buss E.G., Martin R.J., 1982. The developmental characteristics of two strains of chickens selected for differcnces in mature abdominal fat pad size. Growth, 46, 171-181.

LIN C.Y., 1981. Relationship between increased body weight and fat deposition in broilers. World's Poultry Sci. J., 37, 106-110.

Moran E.T. Jr., Summers J.D., OrR H.L., 1968. Back fat, quantitative measure of broiler finish : technique, correlation with grade and effect of dictary caloric density. Food Technol., 22, 999-1002.

Ricard F.H., 1968. Essais d'estimation de l'épaisseur et de l'importance de la peau chez le poulet. Ann. Zootech., 17, 459-466.

RICARD F.H., 1972. Etude de la composition anatomique du poulet. IV. Possibilités d'estimation des pourcentages de viande, d'os et de peau à partir d'une dissection simplifiée des membres. Ann. Zootech., 21, 49-57. 
RiCARD F.H., 1974. Etude de la variabilité génétique de quelques caractéristiques de

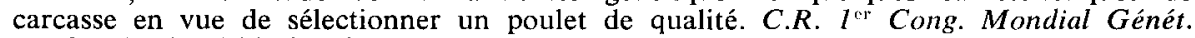
Appliquée, Madrid, 931-940.

RiCard F.H., 1983. Mesure de l'état d'engraissement chez le poulet. Variabilité d'origine biologique .In «Qualité des viandes de volailles», Lahellec, Richard et Colin Ed., Sta. Exp. Avic. Ploufragan, 49-68.

Ricard F.H., Leclerce B., Touraille C., 1983. Selecting broilers for low or high abdominal fat : distribution of carcass fat and quality of meat. Br. Poultry Sci, 24, 511-516.

Scheele C.W., Van Schagen P.J.W., Ten Have H.G.M., 1981. Abdominal and total fat content of three broiler strains at two ages affected by nutritional factors. In "Quality of poultry meat», Mulder, Scheele and Verkamp Editors, Spelderholt Inst. Poultry Res., Beekbergen, 397-407.

Thery C., 1983. La génétique de l'engraissement chez la dinde. Thèse Fac. Sci. Orsay, Université Paris-Sud.

Touchburn S., Simon J., Leclerce B., 1981. Evidence of a glucose-insulin imbalance and effect of dietary protein and energy level in chickens selected for high abdominal fat content. J. Nutr., 111, 325-335. 